\title{
NON-HYPERBOLIC BOUNDARY EQUILIBRIUM BIFURCATIONS IN PLANAR FILIPPOV SYSTEMS: A CASE STUDY APPROACH
}

\author{
MARIO DI BERNARDO, DANIEL J. PAGANO AND ENRIQUE PONCE
}

\begin{abstract}
Boundary equilibrium bifurcations in piecewise smooth discontinuous systems are characterized by the collision of an equilibrium point with the discontinuity surface. Generically, these bifurcations are of codimension one, but there are scenarios where the phenomenon can be of higher codimension. Here, the possible collision of a non-hyperbolic equilibrium with the boundary in a twoparameter framework and the nonlinear phenomena associated with such collision are considered.

By dealing with planar discontinuous (Filippov) systems, some of such phenomena are pointed out through specific representative cases. A methodology for obtaining the corresponding bi-parametric bifurcation sets is developed.
\end{abstract}

\section{INTRODUCTION}

Piecewise smooth dynamical systems are naturally used to model a great variety of engineering devices, which are discontinuous on a macroscopic scale. Examples of such systems include walking mechanisms (Garcia, Chatterjee, Ruina and Coleman, 1998), DC/DC converters (Banerjee and Verghese, 2001) and mechanical systems with impacts and/or friction (Brogliato, 1999; Brogliato, 2000). The corresponding mathematical models are sets of ordinary differential equations (ODEs) characterized by having some discontinuity either in the derivatives of the vector field or in the vector field itself. These discontinuities appear only in a small subset of the phase space and frequently such subset is a smooth manifold, or switching manifold, which is generically transversal to the vector field.

Discontinuous differential systems, also called Filippov systems (Filippov, 1988), form an important class which have been recently studied, focussing on codimension-one bifurcations (both for equilibria and closed orbits) and two-parameter bifurcations of limit cycles. Filippov systems are sets of ODEs with discontinuous right-hand side. It has been shown that these systems can exhibit so-called sliding solutions. Namely, it is possible to 
identify regions of the switching manifold such that the vector field points towards the manifold itself from both of its sides. Then, the system trajectory is constrained to evolve (or slide) on the boundary itself until the vector field on one or the other side of the manifold changes direction. As shown in (Filippov, 1988), and further detailed below, it is possible to construct an appropriate vector field generating such sliding trajectories as a convex combination of the system vector fields on both sides of the boundary.

Filippov systems have been recently shown to exhibit a novel class of bifurcations, termed as discontinuity-induced bifurcations (DIBs), because of their discontinuous nature (di Bernardo, Budd, Champneys, Kowalczyk, Nordmark, Olivar and Piiroinen, 2006). In particular, it was observed that these systems undergo dramatic transitions whenever one of their invariant sets (equilibria, limit cycles etc) interacts non-trivially with the switching manifold in phase space. A striking example is the grazing-sliding bifurcation which was studied in (di Bernardo, Johansson and Vasca, 2001; di Bernardo, Kowalczyk and Nordmark, 2002). There, a limit cycle gains a tangential intersection with the manifold at some parameter value. Then, it can be shown that in many situations the cycle does not persist giving rise to more complex behavior including chaos. Another example is that of boundary equilibrium bifurcations (BEBs) where, under parameter variation, the collision is observed of an equilibrium point with the discontinuity surface. This class of bifurcations was recently studied in (di Bernardo, Nordmark and Olivar, 2007), where attention is focussed on one-parameter BEBs.

Many engineering systems are characterized by more than one parameter. A basic mechanical system, for example, has at least three parameters (mass, friction coefficient and damping). Therefore, it is becoming increasingly apparent that higher-codimension DIBs need to be investigated to gain a deeper understanding of the bifurcation behavior detected experimentally in a growing number of real-world applications. Attempts have been made in the literature to classify and study some two-parameter DIBs, see for example the results in (Cunha, Pagano and Moreno, 2003) and (Kowalczyk, di Bernardo, Champneys, Hogan, Homer, Kuznetsov, Nordmark and Piiroinen, 2006). While attention has mostly focussed to DIBs of limit cycles, little is known on two-parameter boundary equilibrium bifurcations.

The main aim of this paper is to start filling in this gap in the literature by illustrating some of the possible features of two-parameter BEBs. The idea is to analyze in detail a number of simple case studies undergoing a twoparameter BEB. In particular, we study a set of cases where the equilibrium colliding with the discontinuity surface is non-hyperbolic. We study the case of such equilibrium having a null real eigenvalue at the BEB (boundary-fold 
bifurcation) or a pair of complex conjugate eigenvalues (boundary-center and boundary-Hopf).

We present the unfolding of the two-parameter BEB for each of the planar case studies presented in the paper, showing that the local bifurcation diagram is characterized by the presence of bifurcation curves corresponding to both smooth and discontinuity-induced bifurcations. In so doing, we present a new interpretation of the conditions to classify one-parameter BEB in Filippov systems. Also, we illustrate a simple methodology to construct the local bifurcation diagrams in the neighborhood of a two-parameter BEB. It is worth mentioning here that such methodology can be easily extended to cope with higher dimensional problems.

The rest of the paper can be outlined as follows. In Sec. 2 we give a brief overview of the systems of our concern and define the different types of equilibria which are dealt with in the paper. Then, in Sec. 3, we introduce a classification strategy for one-parameter BEBs which is then applied to each of the case studies discussed in the paper. The methodology is described in detail in Sec. 4. Then, we consider three case studies in Sec. 5. The boundary-fold bifurcation of a non-hyperbolic equilibrium is studied in Sec. 5.1. Namely, we assume that at the boundary equilibrium bifurcation, the non-hyperbolic equilibrium also undergoes a smooth fold and present the unfolding of the corresponding two-parameter BEB. The case of a linear centre colliding with the boundary is discussed in Sec. 5.2, where the boundary-centre bifurcation is unfolded. Finally, the simultaneous occurrence of a Hopf bifurcation and a BEB (boundary-Hopf) is discussed in Sec. 5.3. Conclusions are drawn in Sec. 6.

\section{FILIPPOV SYSTEMS, ORDINARY EQUILIBRIA, PSEUDO-EQUILIBRIA AND BOUNDARY EQUILIBRIUM POINTS}

To start with, let us review some elementary facts about Filippov systems. We will adopt the simplest setting which is general enough for applications, without intending to cope with all possible situations. Our main reference will be the work of Kuznetsov et al. (Kuznetsov, Rinaldi and Gragnani, 2003) and we restrict ourselves to consider only autonomous vector fields, with particular attention to equilibria and their possible bifurcations. Suppose that we are given two smooth vector fields $\mathbf{f}^{(i)}: \mathbb{R}^{n} \rightarrow \mathbb{R}^{n}$, $i=1,2$, and a smooth non-constant scalar function $h: \mathbb{R}^{n} \rightarrow \mathbb{R}$ such that the discontinuity manifold

$$
\Sigma=\left\{\mathbf{x} \in \mathbb{R}^{n}: h(\mathbf{x})=0\right\}
$$

is sufficiently regular and generically transversal to both vector fields. Namely, the scalar products $\left\langle\nabla h, \mathbf{f}^{(i)}\right\rangle$, for $i=1,2$, giving each normal 
component of the vector fields with respect to the manifold, are not identically zero on $\Sigma$ (even if they could vanish in some points of the manifold). Let us define the two open regions $S_{1}=\left\{\mathbf{x} \in \mathbb{R}^{n}: h(\mathbf{x})<0\right\}$ and $S_{2}=\left\{\mathbf{x} \in \mathbb{R}^{n}: h(\mathbf{x})>0\right\}$, and the Filippov vector field

$$
F: \mathbb{R}^{n} \backslash \Sigma \rightarrow \mathbb{R}^{n}, \text { such that } F(\mathbf{x})=\left\{\begin{array}{l}
\mathbf{f}^{(1)}(\mathbf{x}), \text { if } \mathbf{x} \in S_{1} \\
\mathbf{f}^{(2)}(\mathbf{x}), \text { if } \mathbf{x} \in S_{2}
\end{array}\right.
$$

Then, in general the resulting vector field cannot be continuously extended to the manifold $\Sigma$ and some procedure must be designed in order to define appropriately the solutions of the differential system

$$
\dot{\mathbf{x}}=F(\mathbf{x}) .
$$

Take a point $\hat{\mathbf{x}} \in \Sigma$ and assume that $\left\langle\nabla h(\hat{\mathbf{x}}), \mathbf{f}^{(1)}(\hat{\mathbf{x}})\right\rangle>0$. Then, such a point is reachable for some orbit of the system $\dot{\mathbf{x}}=\mathbf{f}^{(1)}(x)$ with initial condition within $S_{1}$. If furthermore it turns out that we also have $\left\langle\nabla h(\hat{\mathbf{x}}), \mathbf{f}^{(2)}(\hat{\mathbf{x}})\right\rangle>0$, it seems natural to concatenate the orbit of $\dot{x}=$ $\mathbf{f}^{(1)}(x)$ finishing at $\hat{\mathbf{x}}$ with the orbit of $\dot{\mathbf{x}}=\mathbf{f}^{(2)}(\mathbf{x})$ starting at the same point. We can adopt the same reasoning in the analogous case, when both $\left\langle\nabla h(\hat{\mathbf{x}}), \mathbf{f}^{(2)}(\hat{\mathbf{x}})\right\rangle<0$ and $\left\langle\nabla h(\hat{\mathbf{x}}), \mathbf{f}^{(1)}(\hat{\mathbf{x}})\right\rangle<0$, and define the crossing set (sometimes called the sewing part of $\Sigma$ ) as

$$
\Sigma_{c}=\left\{\mathbf{x} \in \Sigma:\left\langle\nabla h(\mathbf{x}), \mathbf{f}^{(1)}(\mathbf{x})\right\rangle \cdot\left\langle\nabla h(\mathbf{x}), \mathbf{f}^{(2)}(\mathbf{x})\right\rangle>0\right\} \subset \Sigma .
$$

In points belonging to $\Sigma_{c}$, standard solutions of the two systems can be joined to form a solution whose orbit crosses the discontinuity manifold. Note that, apart from the above geometrical interpretation on the pointing directions of the vector fields in points of the discontinuity surface, one can also think of the instantaneous change rate for the value of $h$ along a solution $\mathbf{x}(t)$, and conclude that

$$
\frac{d}{d t}[h(\mathbf{x}(t))]=\nabla h(\mathbf{x}(t)) \cdot \frac{d}{d t} \mathbf{x}(t)=\left\langle\nabla h(\mathbf{x}(t)), \mathbf{f}^{(i)}(\mathbf{x}(t))\right\rangle,
$$

with $i=1$ or 2 , depending on the corresponding vector field associated to the solution considered.

According to Kuznetsov et al. (Kuznetsov et al., 2003), we can define the non-crossing part of $\Sigma$, or sliding part, as the complement to $\Sigma_{c}$ in $\Sigma$, that is,

$$
\Sigma_{s}=\left\{\mathbf{x} \in \Sigma:\left\langle\nabla h(\mathbf{x}), \mathbf{f}^{(1)}(\mathbf{x})\right\rangle \cdot\left\langle\nabla h(\mathbf{x}), \mathbf{f}^{(2)}(\mathbf{x})\right\rangle \leq 0\right\} .
$$


Thus, while the crossing set is an open set, the sliding set should be the union of closed subsets (some of them possibly consisting of isolated sliding points) $)^{1}$.

Within the sliding set, the Filippov method can be used to construct solutions, to be considered as extensions for solutions of (2). Such a method consists in defining a new vector field computed from an adequate convex combination of the two original vector fields, namely

$$
F_{s}(\mathbf{x})=(1-\lambda) \mathbf{f}^{(1)}(\mathbf{x})+\lambda \mathbf{f}^{(2)}(\mathbf{x}),
$$

where for each $\mathbf{x} \in \Sigma_{s}$ the value of $\lambda$ is selected such that $\left\langle\nabla h(\mathbf{x}), F_{s}(\mathbf{x})\right\rangle=$ 0 . A simple computation shows that

$$
\lambda=\lambda(\mathbf{x})=\frac{\left\langle\nabla h(\mathbf{x}), \mathbf{f}^{(1)}(\mathbf{x})\right\rangle}{\left\langle\nabla h(\mathbf{x}), \mathbf{f}^{(1)}(\mathbf{x})-\mathbf{f}^{(2)}(\mathbf{x})\right\rangle},
$$

provided the above denominator does not vanish and then, by using the definition of $\Sigma_{s}$, one concludes that

$$
0 \leq \lambda(\mathbf{x}) \leq 1, \quad \text { for all } \mathbf{x} \in \Sigma_{s}
$$

Therefore, we have a explicit definition for the sliding vector field, namely

$$
F_{s}(\mathbf{x})=\frac{\left\langle\nabla h(\mathbf{x}), \mathbf{f}^{(2)}(\mathbf{x})\right\rangle \mathbf{f}^{(1)}(\mathbf{x})-\left\langle\nabla h(\mathbf{x}), \mathbf{f}^{(1)}(\mathbf{x})\right\rangle \mathbf{f}^{(2)}(\mathbf{x})}{\left\langle\nabla h(\mathbf{x}), \mathbf{f}^{(2)}(\mathbf{x})-\mathbf{f}^{(1)}(\mathbf{x})\right\rangle} .
$$

If $\left\langle\nabla h(\mathbf{x}), \mathbf{f}^{(2)}(\mathbf{x})-\mathbf{f}^{(1)}(\mathbf{x})\right\rangle=0$ for some $\mathbf{x} \in \Sigma_{s}$, then we say that such $\mathrm{x}$ is a singular sliding point. This can happen in three cases: both vector fields are tangent to $\Sigma$; one is tangent and the other vanishes; both vector fields vanish at the point. In all these singular cases, if we exclude infinitely degenerate cases and the sliding point is non-isolated, it is possible to define the vector field $F_{s}$ by continuity arguments. For isolated singular sliding points it will be taken $F_{s}(\mathbf{x})=0$. Clearly, the boundary of the sliding set $\Sigma_{s}$ (as a subset of $\Sigma$ ) is associated to each one of the two equalities $\left\langle\nabla h(\mathbf{x}), \mathbf{f}^{(1)}(\mathbf{x})\right\rangle=0,\left\langle\nabla h(\mathbf{x}), \mathbf{f}^{(2)}(\mathbf{x})\right\rangle=0$; that is, $\lambda(\mathbf{x})=0$ or $\lambda(\mathbf{x})=1$ with $\mathbf{x} \in \Sigma$.

As usual in the analysis of dynamical systems, we must look for the simplest solutions organizing the dynamics, namely the constant solutions associated to rest points normally called equilibria. Of course, the Filippov

\footnotetext{
${ }^{1}$ We must mention that some authors prefer to qualify as sliding only the case when $\left\langle\nabla h(x), \mathbf{f}^{(2)}(x)\right\rangle<0<\left\langle\nabla h(x), \mathbf{f}^{(1)}(x)\right\rangle$

(to be called here attractive sliding case) and, otherwise, that is if$$
\left\langle\nabla h(x), \mathbf{f}^{(1)}(x)\right\rangle \leq 0 \leq\left\langle\nabla h(x), \mathbf{f}^{(2)}(x)\right\rangle,
$$

then they speak of the escaping part of $\Sigma$.
} 
system (2) inherit the equilibria of each vector field $\mathbf{f}^{i}(\mathbf{x})$, but we must be cautious and distinguish between real or virtual equilibria. In particular, we call

- admissible or real equilibrium points to all the solutions of $\mathbf{f}^{(1)}(\mathbf{x})=$ 0 that belong to $S_{1}$ and the solutions of $\mathbf{f}^{(2)}(\mathbf{x})=0$ that belong to $S_{2}$, while

- virtual equilibrium points are the solutions of $\mathbf{f}^{(1)}(\mathbf{x})=0$ that belong to $S_{2}$, and the solutions of $\mathbf{f}^{(2)}(\mathbf{x})=0$ that belong to $S_{1}$.

Although virtual equilibria are not equilibria of $F(x)$, they can still organize the dynamics in the corresponding region. Regarding now the induced dynamical system $\dot{\mathbf{x}}=F_{s}(\mathbf{x})$ on the sliding set, we note that apart from isolated singular sliding points, the sliding vector field $F_{s}$ can vanish in other points which behave like real equilibria for such dynamical system if we restrict our attention to the set $\Sigma_{s}$. They also are, in some sense, equilibria for system (2) and will be called pseudo-equilibrium points. For instance, when both vectors $\mathbf{f}^{(i)}$ are transversal to $\Sigma$ in a certain point of this surface and furthermore they are anti-collinear, that is, there exist $\lambda_{1}, \lambda_{2}>0$, such that

$$
\lambda_{1} \mathbf{f}^{(1)}(\mathbf{x})+\lambda_{2} \mathbf{f}^{(2)}(\mathbf{x})=0,
$$

then a simple argument shows that the point is necessarily in $\Sigma_{s}$, since the value of $\left\langle\nabla h(\mathbf{x}), \mathbf{f}^{(i)}(\mathbf{x})\right\rangle, i=1,2$ is non-zero and with different sign. In fact, it is immediate to conclude that at such point one has $F_{s}(\mathbf{x})=0$ and so it is a pseudo-equilibrium for (2). Reciprocally, if $\mathbf{x}$ is a point in $\Sigma_{s}$ with $F_{s}(\mathbf{x})=0$ and it is not a tangency point, we easily conclude that both vector fields are anti-collinear at the point. If for instance at such point we assume $\left\langle\nabla h(\mathbf{x}), \mathbf{f}^{(2)}(\mathbf{x})\right\rangle<0<\left\langle\nabla h(\mathbf{x}), \mathbf{f}^{(1)}(\mathbf{x})\right\rangle$, we conclude that two orbits, each one corresponding to a vector field, collide with opposite directions and determine a rest point for the global vector field. This rest point, differently from what observed for admissible equilibria, can be reached in finite time from points belonging to these two orbits.

A general and unified necessary condition for equilibria, to be called Generalized Condition for Equilibria (GCE), can be established from Eq. (6). Effectively, the condition $F_{s}(\mathbf{x})=0$ translates to

$$
\left\langle\nabla h(\mathbf{x}), \mathbf{f}^{(2)}(\mathbf{x})\right\rangle \mathbf{f}^{(1)}(\mathbf{x})-\left\langle\nabla h(\mathbf{x}), \mathbf{f}^{(1)}(\mathbf{x})\right\rangle \mathbf{f}^{(2)}(\mathbf{x})=0
$$

and it is obvious that this condition is also satisfied by virtual and admissible equilibria.

To end this section, an important remark is that $F_{s}(\mathbf{x})=0$ when any of the vector fields vanishes at a point of $\Sigma$. Then we define such a point as a boundary equilibrium point. Thus, a point $\mathbf{x}$ is a boundary equilibrium point 
if $\mathbf{f}^{(1)}(\mathbf{x})=0$ or $\mathbf{f}^{(2)}(\mathbf{x})=0$ and $h(\mathbf{x})=0$. On the one hand, a boundary equilibrium point can be seen as the critical case between admissible and virtual equilibria. On the other hand, if we exclude the exceptional case of being a singular sliding point, we deduce from (5) that either $\lambda(\mathbf{x})=0$ or $\lambda(\mathbf{x})=1$, that is, the point is also at the boundary of the sliding set $\Sigma_{s}$.

\section{BOUNDARY EQUILIBRIUM BIFURCATIONS}

We will now discuss the occurrence of boundary equilibrium bifurcations in Filippov systems. We suppose that the two defining vector fields and the manifold determining function $h$ depend on some real parameter $\mu \in \mathbb{R}$. Also, we assume that for a critical value of the parameter, say $\mu=0$, there appears a boundary equilibrium point associated to the first vector field $\mathbf{f}^{(1)}$. Without loss of generality, we assume that the point is at the origin, that is, $(\mathbf{x}, \mu)=(0,0)$ is a solution of the equations

$$
\begin{array}{r}
\mathbf{f}^{(1)}(\mathbf{x}, \mu)=0, \\
h(\mathbf{x}, \mu)=0,
\end{array}
$$

and the corresponding solution branch $\overline{\mathbf{x}}(\mu)$, with $\overline{\mathbf{x}}(0)=0$, is regular, that is,

$$
A=D_{\mathbf{x}} \mathbf{f}^{(1)}(0,0) \text { is invertible. }
$$

If we also assume that the branch is transversal to $\Sigma$, namely,

$$
\left.\frac{d}{d \mu} h(\overline{\mathbf{x}}(\mu), \mu)\right|_{\mu=0} \neq 0
$$

a natural question arises. How does the situation change in passing the parameter through the critical value $\mu=0$ ?

Let us first write in a explicit way the transversality condition. For that, we apply the implicit function theorem (I.F.T. for short, in the sequel) to the first equation in (9) by differentiating with respect to $\mu$, to get

$$
D_{\mathbf{x}} \mathbf{f}^{(1)}(\overline{\mathbf{x}}(\mu), \mu) \frac{d}{d \mu} \overline{\mathbf{x}}(\mu)+D_{\mu} \mathbf{f}^{(1)}(\overline{\mathbf{x}}(\mu), \mu)=0,
$$

and, by considering $\mu=0$, after defining $M=D_{\mu} \mathbf{f}^{(1)}(0,0)$, we obtain

$$
\overline{\mathbf{x}}^{\prime}(0)=\left.\frac{d}{d \mu} \overline{\mathbf{x}}(\mu)\right|_{\mu=0}=-A^{-1} M
$$

Then, now

$$
\frac{d}{d \mu} h(\overline{\mathbf{x}}(\mu), \mu)=D_{\mathbf{x}} h(\overline{\mathbf{x}}(\mu), \mu) \frac{d}{d \mu} \overline{\mathbf{x}}(\mu)+D_{\mu} \mathbf{f}^{(1)}(\overline{\mathbf{x}}(\mu), \mu),
$$


and defining $C^{T}=D_{\mathbf{x}} h(0,0), N=D_{\mu} h(0,0)$, the transversality condition reduces to

$$
\left.\frac{d}{d \mu} h(\overline{\mathbf{x}}(\mu), \mu)\right|_{\mu=0}=N-C^{T} A^{-1} M \neq 0 .
$$

It should be noticed that for $\mu=0$, the origin is also an equilibrium point for the sliding vector field (4) corresponding to the value $\lambda=0$, that is, it is in one of the two boundaries of the sliding set $\Sigma_{s}$. Thus, coming back to the pending question, such equilibrium could be also a point of a solution $\operatorname{branch}(\tilde{\mathbf{x}}(\mu), \tilde{\lambda}(\mu))$, with $(\tilde{\mathbf{x}}(0), \tilde{\lambda}(0))=(0,0)$, for the system of equations

$$
\begin{aligned}
(1-\lambda) \mathbf{f}^{(1)}(\mathbf{x}, \mu)+\lambda \mathbf{f}^{(2)}(\mathbf{x}, \mu) & =0, \\
h(\mathbf{x}, \mu) & =0 .
\end{aligned}
$$

Such a solution branch for the sliding vector field will be locally regular when the hypotheses of I.F.T. are fulfilled in order to solve the above system for $(\mathbf{x}, \lambda)$ in terms of $\mu$, near the point $(\mathbf{x}, \lambda, \mu)=(0,0,0)$. It then suffices that in the following system of equations, obtained by implicit differentiation with respect to $\mu$, namely

$$
\begin{aligned}
& {\left[\begin{array}{cc}
(1-\lambda) D_{\mathbf{x}} \mathbf{f}^{(1)}(\mathbf{x}, \mu)+\lambda D_{\mathbf{x}} \mathbf{f}^{(2)}(\mathbf{x}, \mu) & \mathbf{f}^{(2)}(\mathbf{x}, \mu)-\mathbf{f}^{(1)}(\mathbf{x}, \mu) \\
D_{\mathbf{x}} h(\mathbf{x}, \mu) & 0
\end{array}\right]\left[\begin{array}{c}
\mathbf{x}^{\prime}(\mu) \\
\lambda^{\prime}(\mu)
\end{array}\right]+} \\
& (11) \quad+\left[\begin{array}{c}
(1-\lambda) D_{\mu} \mathbf{f}^{(1)}(\mathbf{x}, \mu)+\lambda D_{\mu} \mathbf{f}^{(2)}(\mathbf{x}, \mu) \\
D_{\mu} h(\mathbf{x}, \mu)
\end{array}\right]=\left[\begin{array}{l}
0 \\
0
\end{array}\right]
\end{aligned}
$$

the matrix of coefficients be non-singular at $(\mathbf{x}, \lambda, \mu)=(0,0,0)$. Taking into account that $\mathbf{f}^{(1)}(0,0)=0$ by our initial hypothesis, and introducing the vector $B=\mathbf{f}^{(2)}(0,0)$, the condition can be written as

$$
\operatorname{det}\left[\begin{array}{cc}
A & B \\
C^{T} & 0
\end{array}\right] \neq 0
$$

Using the equality

$$
\left[\begin{array}{cc}
A^{-1} & 0 \\
C^{T} A^{-1} & -1
\end{array}\right]\left[\begin{array}{cc}
A & B \\
C^{T} & 0
\end{array}\right]=\left[\begin{array}{cc}
I & A^{-1} B \\
0 & C^{T} A^{-1} B
\end{array}\right]
$$

we see that

$$
\operatorname{det}\left[\begin{array}{cc}
A & B \\
C^{T} & 0
\end{array}\right]=-C^{T} A^{-1} B \cdot \operatorname{det} A,
$$

and our conclusion is that if $C^{T} A^{-1} B \neq 0$, there exists locally a unique equilibrium branch $(\tilde{\mathbf{x}}(\mu), \tilde{\lambda}(\mu))$, with $(\tilde{\mathbf{x}}(0), \tilde{\lambda}(0))=(0,0)$ for the sliding 
vector field which will be admissible whenever $0 \leq \tilde{\lambda}(\mu) \leq 1$. Moreover, from (11), using the I.F.T. we have

$$
\begin{aligned}
A \tilde{\mathbf{x}}^{\prime}(0)+B \tilde{\lambda}^{\prime}(0)+M & =0, \\
C^{T} \tilde{\mathbf{x}}^{\prime}(0)+N & =0 .
\end{aligned}
$$

After some standard manipulation and recalling (10), we then have

$$
\tilde{\lambda}^{\prime}(0)=\frac{N-C^{T} A^{-1} M}{C^{T} A^{-1} B}=\left.\frac{1}{C^{T} A^{-1} B} \cdot \frac{d}{d \mu} h(\overline{\mathbf{x}}(\mu), \mu)\right|_{\mu=0} .
$$

Hence, for the two colliding equilibrium branches, we conclude that

$$
\left.\frac{d}{d \mu} \tilde{\lambda}(\mu)\right|_{\mu=0}=\left.\frac{1}{C^{T} A^{-1} B} \cdot \frac{d}{d \mu} h(\overline{\mathbf{x}}(\mu), \mu)\right|_{\mu=0},
$$

so that we can easily prove the following result. Note that the theorem below already appeared in (di Bernardo et al., 2007), but here we provide a more detailed derivation by resorting to the I.F.T.

Theorem 1. Assume that for $\mu=0$ an equilibrium branch of the vector field $\mathbf{f}^{(1)}(\mathbf{x}, \mu)$ crosses the discontinuity manifold $h(\mathbf{x}, \mu)=0$ at the origin, being this point regular, that is, $A=D_{\mathbf{x}} \mathbf{f}^{(1)}(0,0)$ is a non-singular matrix. Furthermore, suppose that the crossing is transversal, that is, if $M=D_{\mu} \mathbf{f}^{(1)}(0,0), C^{T}=D_{\mathbf{x}} h(0,0)$, and $N=D_{\mu} h(0,0)$ then

$$
\left.\frac{d}{d \mu} h(\overline{\mathbf{x}}(\mu), \mu)\right|_{\mu=0}=N-C^{T} A^{-1} M \neq 0,
$$

and the non-degeneracy condition $C^{T} A^{-1} B \neq 0$, where $B=\mathbf{f}^{(2)}(0,0)$, is satisfied. The following statements hold.

(a): (Persistence of one equilibrium for the Filippov system) If $C^{T} A^{-1} B>0$ the equilibrium branch for $\mathbf{f}^{(1)}(\mathbf{x}, \mu)$ and the equilibrium branch for the sliding vector field $F_{s}$ do not coexist for $\mu \neq 0$ and small. More precisely, when $\mu$ varies in a neighborhood of 0 one admissible equilibrium for $\mathbf{f}^{(1)}$ becomes non-admissible for $\mu=0$ and it is transformed into an admissible pseudo-equilibrium, that is, into an admissible equilibrium for the sliding vector field (or vice versa).

(b): (Non-smooth fold or annihilation of equilibria for the Filippov system) When the condition $C^{T} A^{-1} B<0$ holds, the equilibrium branch for $\mathbf{f}^{(1)}(\mathbf{x}, \mu)$ and the equilibrium branch for the sliding vector field $F_{s}$ coexist for small values de $\mu$, but only either for $\mu<0$ or for $\mu>0$. That is, when $\mu$ varies in a neighborhood of 0 one admissible equilibrium for $\mathbf{f}^{(1)}$ collides for $\mu=0$ with one admissible pseudo-equilibrium and both become non-admissible. 
Proof. Under the given hypotheses, the I.F.T. assures local existence and uniqueness of the two solution branches involved. Suppose, for instance, that $C^{T} A^{-1} B>0$. If $N-C^{T} A^{-1} M>0$, then we know that for small $\mu>0$ the equilibrium of $\mathbf{f}^{(1)}(\mathbf{x}, \mu)$ becomes non-admissible, since

$$
\left.\frac{d}{d \mu} h(\overline{\mathbf{x}}(\mu), \mu)\right|_{\mu=0}>0
$$

and then $h(\overline{\mathbf{x}}(\mu), \mu)$ is an increasing function with $h(\overline{\mathbf{x}}(0), 0)=0$. From the assumptions, we also know that

$$
\left.\frac{d}{d \mu} \tilde{\lambda}(\mu)\right|_{\mu=0}>0,
$$

so that we conclude that $\tilde{\lambda}(\mu)>0$ for small $\mu>0$, and so the pseudoequilibrium is then admissible, going to the interior of the sliding part (it was non-admissible for $\mu<0$ ).

All the remaining cases can be shown in the same way, and the conclusion follows

Note that if the vector field of reference is not $\mathbf{f}^{(1)}$, but instead the one with $h(\mathbf{x})>0$, that is, the equilibrium branch colliding with the discontinuity surface is a zero of $\mathbf{f}^{(2)}$, then from (4) we also get a zero of the sliding vector field $F_{s}$, but now corresponding to $\lambda=1$. Thus, to apply the above result you must define accordingly $A=D_{\mathbf{x}} \mathbf{f}^{(2)}(0,0), M=D_{\mu} \mathbf{f}^{(2)}(0,0)$, $N=D_{\mu} \mathbf{f}^{(2)}(0,0)$ and then $B=\mathbf{f}^{(1)}(0,0)$. Repeating the computations, we now apply the I.F.T. at $(\mathbf{x}, \lambda, \mu)=(0,1,0)$ getting the same expressions if we write $-B$ instead of $B$. This implies that you get the opposite characterization, and for instance the condition $C^{T} A^{-1} B>0$ now corresponds to the non-smooth fold case.

\section{A Methodology for STUdying NON-HyPeRBolic BEBs}

The concepts and results reviewed in Sections 2 and 3 have been developed in a one-parameter context, where the parameter affected both the vector fields and the function defining the discontinuity manifold. Now to pave the way for the analysis to be made in the following sections, which naturally requires a two-parameter framework, we will first indicate the methodology to be used in all the cases to be dealt with. It is worth mentioning here that such a methodology should be also useful in many other situations not considered in this work. We restrict our attention in what follows to planar cases, so that avoiding as much subscripts as possible for ease of reading, we use for coordinates in $\mathbb{R}^{2}$ the notation $\mathbf{x}=(x, y)$. 
As indicated before, we want to analyze the nonlinear phenomena associated to the possible collision, by moving parameters, of a possibly nonhyperbolic equilibrium point with a discontinuity surface. Although the general situation could be different, it is not very restrictive to assume the existence of only two parameters playing separated roles, and so we adopt the following scheme. One of the two parameters is to be responsible at some critical value for the eventual collision of the equilibrium point with the discontinuity manifold and, independently, the other parameter will control the possible lack of hyperbolicity of the equilibrium.

Furthermore, and without loss of generality, we may suppose that the equilibrium point is fixed at the origin and that it is in fact the movement of the discontinuity manifold which causes the collision between the equilibrium and the manifold. This point of view allows us to distribute the two parameters in such a way that the 'collision' parameter only affects the definition of the manifold while the 'hyperbolicity' parameter only appears in the definition of the vector field, being compatible with the approach followed in previous section.

Thus, let us define the two open regions

$$
\begin{aligned}
& S_{1}=S_{1}(\rho)=\left\{\mathbf{x} \in \mathbb{R}^{2}: h(x, y, \rho)<0\right\}, \\
& S_{2}=S_{2}(\rho)=\left\{\mathbf{x} \in \mathbb{R}^{2}: h(x, y, \rho)>0\right\},
\end{aligned}
$$

where $\Sigma=\Sigma(\rho)=\left\{\mathbf{x} \in \mathbb{R}^{2}: h(x, y, \rho)=0\right\}$ gives the boundary between $S_{1}$ and $S_{2}$, and when needed we will emphasize the dependence of such sets on the parameter $\rho$. For the Filippov vector field $F:\left(\mathbb{R}^{2} \backslash \Sigma\right) \times \mathbb{R} \rightarrow \mathbb{R}^{2}$, such that

$$
F(x, y, \nu)= \begin{cases}\mathbf{f}^{(1)}(x, y, \nu), & (x, y) \in S_{1}(\rho) \\ \mathbf{f}^{(2)}(x, y, \nu), & (x, y) \in S_{2}(\rho)\end{cases}
$$

we will assume that the origin is for $\nu=0$ a non-hyperbolic point for $\mathbf{f}^{(1)}$, and that such point hits transversally the discontinuity boundary $\Sigma(\rho)$ for the critical value $\rho=0$. Our aim is to describe in the parameter plane $(\rho, \nu) \in \mathbb{R}^{2}$, the bifurcation set to be expected in some canonical cases. Note that according to our scheme the dependence of $\mathbf{f}^{(2)}$ on the parameter $\nu$ is not strictly required; in fact, it should be better that this vector field have not equilibria near the origin in order not to contaminate the results, at least locally speaking. Also, there is no loss of generality if we assume that $\Sigma(\rho)$ is a vertical line which moves with $\rho$, that is we can deal with the simple case $h(x, y, \rho)=x-\rho$. 
Adopting the standard notation for the several components of the vector fields, namely

$$
\mathbf{f}^{(1)}(x, y, \nu)=\left[\begin{array}{c}
f_{1}^{(1)}(x, y, \nu) \\
f_{2}^{(1)}(x, y, \nu)
\end{array}\right] ; \quad \mathbf{f}^{(2)}(x, y, \nu)=\left[\begin{array}{c}
f_{1}^{(2)}(x, y, \nu) \\
f_{2}^{(2)}(x, y, \nu)
\end{array}\right],
$$

we will carry on the analysis of Filippov systems, by using the following methodology.

(1) First, admissible and virtual equilibria of system (13) will be determined by solving the equations

$$
\mathbf{f}^{(1)}(x, y, \nu)=0, \quad \mathbf{f}^{(2)}(x, y, \nu)=0 .
$$

(2) In a second step, we will obtain the sliding region $\Sigma_{s}$ of system (13). Although the discontinuity manifold only depends on one parameter, note that in general, $\Sigma_{s}$ will depend on both parameters $\rho, \nu$. Using the notation $\nabla h(x, y, \rho)=\left[h_{x}(x, y, \rho), h_{y}(x, y, \rho)\right]$, Eq. (3) can be explicitly written as

$$
\left(h_{x} \cdot f_{1}^{(1)}+h_{y} \cdot f_{2}^{(1)}\right)\left(h_{x} \cdot f_{1}^{(2)}+h_{y} \cdot f_{2}^{(2)}\right) \leq 0,
$$

where the arguments are not explicit for brevity. In the particular case where $h(x, y, \rho)=x-\rho$, we have then $\nabla h=[1,0]$, and so

$\Sigma_{s}(\rho, \nu)=\left\{(x, y) \in \mathbb{R}^{2}: x=\rho, \quad f_{1}^{(1)}(\rho, y, \nu) \cdot f_{1}^{(2)}(\rho, y, \nu) \leq 0\right\}$,

which graphically can be easily determined by drawing the vertical nullclines of both vector fields.

(3) Third, pseudo-equilibria of system (13) are determined as follows. For the GCE given in Eq. (8), we have that its first and second components becomes

$$
\begin{aligned}
& \left(h_{x} \cdot f_{1}^{(2)}+h_{y} \cdot f_{2}^{(2)}\right) f_{1}^{(1)}-\left(h_{x} \cdot f_{1}^{(1)}+h_{y} \cdot f_{2}^{(1)}\right) f_{1}^{(2)}=0, \\
& \left(h_{x} \cdot f_{1}^{(2)}+h_{y} \cdot f_{2}^{(2)}\right) f_{2}^{(1)}-\left(h_{x} \cdot f_{1}^{(1)}+h_{y} \cdot f_{2}^{(1)}\right) f_{2}^{(2)}=0,
\end{aligned}
$$

which simplifies to

$$
\begin{aligned}
& h_{y}(x, y, \rho)\left(f_{1}^{(1)}(x, y, \nu) \cdot f_{2}^{(2)}(x, y, \nu)-f_{2}^{(1)}(x, y, \nu) \cdot f_{1}^{(2)}(x, y, \nu)\right)=0, \\
& h_{x}(x, y, \rho)\left(f_{1}^{(2)}(x, y, \nu) \cdot f_{2}^{(1)}(x, y, \nu)-f_{1}^{(1)}(x, y, \nu) \cdot f_{2}^{(2)}(x, y, \nu)\right)=0 .
\end{aligned}
$$

Once more, we see that in any regular point of $\Sigma_{s}$, that is if $h_{x}$ and $h_{y}$ are both non-zero, the pseudo-equilibria condition reduces to the collinearity condition

$$
f_{1}^{(1)}(x, y, \nu) \cdot f_{2}^{(2)}(x, y, \nu)-f_{2}^{(1)}(x, y, \nu) \cdot f_{1}^{(2)}(x, y, \nu)=0 .
$$


(4) The analysis of the BEB point will be carried out using the strategy presented in Section 3. In our case, as $h(x, y, \rho)=x-\rho$ and we want to consider the situation where a non-hyperbolic equilibrium for $\mathbf{f}^{(1)}$ is at the origin, we must anticipate a boundary bifurcation for $\rho=0$ for certain values of $\nu$. Thus, the parameter $\rho$ will play the role of the bifurcation parameter responsible for the possible collision of two equilibrium branches.

\section{Case Studies}

In what follows, using the steps listed above, we consider the boundary equilibrium bifurcation of some representative non-hyperbolic situations. In particular, we investigate the dynamics of a set of exemplary Filippov systems obtained by:

- Case Study 1: switching between the saddle-node normal form and a constant attracting vector field.

- Case Study 2: switching between a linear center and a constant attracting vector field.

- Case Study 3: switching between the normal form of the Hopf bifurcation and a constant attracting vector field.

A summarizing list of the notation that will be used in the rest of this section to label the various bifurcation curves and invariant sets is given in Tab. 1.

\begin{tabular}{|c|c|}
\hline Acronyms & Meaning \\
\hline $\mathrm{AE}$ & Admissible Equilibrium point \\
$\mathrm{VE}$ & Virtual Equilibrium point \\
$\mathrm{pE}$ & pseudo Equilibrium point \\
$\mathrm{LC}$ & Limit Cycle \\
sLC & Stable Limit Cycle \\
$\mathrm{uLC}$ & Unstable Limit Cycle \\
$\mathrm{H}$ & Hopf bifurcation \\
$\mathrm{G}$ & Grazing bifurcation \\
F-C-LC & Focus-Center-Limit Cycle bifurcation \\
$\mathrm{SNE}$ & Saddle Node of Equilibria \\
$\mathrm{SNpE}$ & Saddle Node of pseudo-Equilibria \\
$\mathrm{HC}$ & Homoclinic Connection \\
$\mathrm{E}_{\mathrm{A}}$ & Equilibria Annihilation or Non-Smooth-Fold \\
$\mathrm{E}_{\mathrm{T}}$ & Equilibria Transition or Persistence \\
$\mathrm{GCE}$ & Generalized Condition for Equilibria \\
\hline
\end{tabular}

TABLE 1. List of acronyms used in the text. 
5.1. Case Study 1: Boundary-fold Bifurcation. Our first example of non-hyperbolic point colliding with the discontinuity surface will be the case corresponding to the saddle-node bifurcation, that is we allow an eigenvalue to be zero. In all the cases, to simplify the expression of the vector field, we assume the manifold $\Sigma$ to be a moving vertical line, that is $h(x, y, \rho)=x-\rho$. Thus, let us consider for $x<\rho$ the vector field

$$
\mathbf{f}^{(1)}(x, y, \nu)=\left[\begin{array}{c}
\nu-x^{2} \\
-y
\end{array}\right]
$$

so that we can have equilibrium points at $(\bar{x}, \bar{y})=( \pm \sqrt{\nu}, 0)$ provided that $\nu \geq 0$. In particular, since the linearization matrix becomes

$$
J(\bar{x}, \bar{y})=\left[\begin{array}{cc}
-2 \bar{x} & 0 \\
0 & -1
\end{array}\right]=\left[\begin{array}{cc}
\mp 2 \sqrt{\nu} & 0 \\
0 & -1
\end{array}\right],
$$

we see that the origin is a non-hyperbolic equilibrium for $\nu=0$, which undergoes a saddle-node bifurcation giving rise to a saddle $(-\sqrt{\nu}, 0)$ and a stable node $(\sqrt{\nu}, 0)$ for $\nu>0$. For $\nu<0$ the vector field $\mathbf{f}^{(1)}$ has no equilibrium at all.

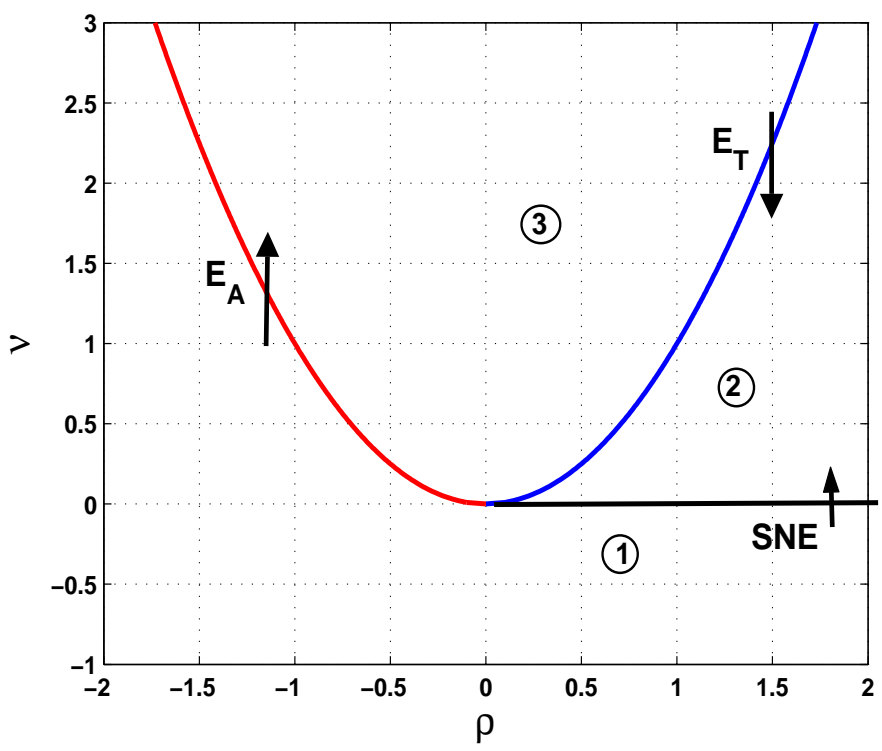

Figure 1. Bifurcation set in the plane $(\rho, \nu)$ for the case of saddle-node plus a constant vector field. 

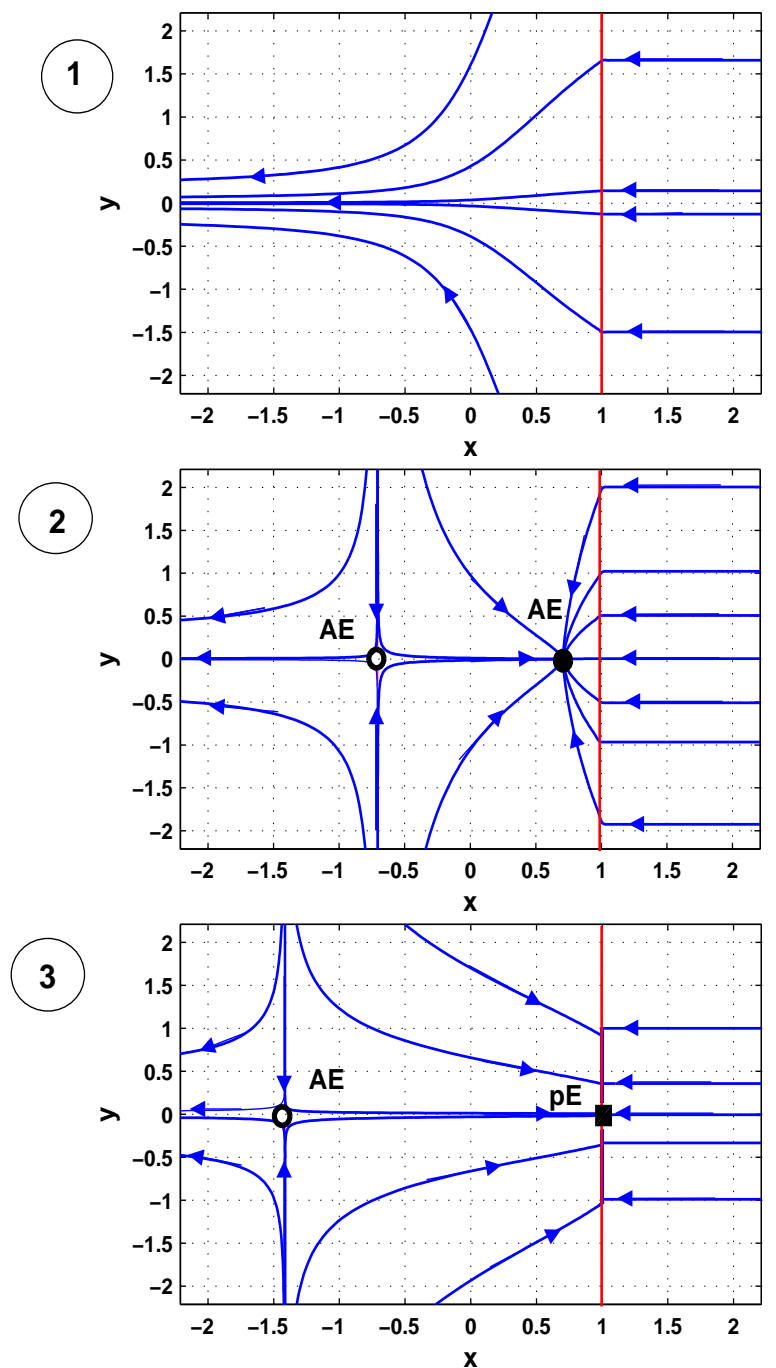

Figure 2. Some state space diagrams in the plane $(x, y)$ for the saddle-node plus a constant vector field case. The three cases correspond to $\rho=1$ but with different values of $\nu=\{-0.5,0.5,2\}$.

As the second vector field involved, now for $x>\rho$, we consider the simplest possible case, that is, a constant horizontal vector field pushing to 
the left, namely

$$
\mathbf{f}^{(2)}(x, y, \nu)=\left[\begin{array}{c}
-1 \\
0
\end{array}\right],
$$

which obviously cannot give rise to any admissible equilibria. Note that this choice also implies that any sliding region will be attractive, and so we will not deal with escaping regions.

The second step in our analysis is to compute the sliding set $\Sigma_{s}$. According to (15), we have the set

$$
\left\{(x, y) \in \mathbb{R}^{2}: x=\rho, \quad(-1)\left(\nu-x^{2}\right)=x^{2}-\nu \leq 0\right\}
$$

that is

$$
\Sigma_{s}=\Sigma_{s}(\rho, \nu)=\left\{\begin{array}{cc}
\left\{(x, y) \in \mathbb{R}^{2}: x=\rho\right\} & \text { if } \nu \geq \rho^{2}, \\
\emptyset & \text { if } \nu<\rho^{2}
\end{array}\right.
$$

so that, when it is non-empty there is no restriction for $y$ and we get $\Sigma_{s}=\Sigma$, that is the full vertical line $x=\rho$.

Assuming $\nu \geq \rho^{2}$, and using (16) we detect a pseudo-equilibrium point in $\Sigma_{s}$ when

$$
\left(\nu-x^{2}\right) \cdot 0-(-y)(-1)=0,
$$

that is $y=0$. Note that, since

$$
\left\langle\nabla h(\mathbf{x}), \mathbf{f}^{(2)}(\mathbf{x})-\mathbf{f}^{(1)}(\mathbf{x})\right\rangle=[1,0]^{T}\left[\begin{array}{c}
x^{2}-\nu-1 \\
y
\end{array}\right]=x^{2}-\nu-1,
$$

according to $(6)$ we get

$$
F_{s}(\mathbf{x})=\frac{1}{\rho^{2}-\nu-1}\left[(-1)\left(\begin{array}{c}
\nu-\rho^{2} \\
-y
\end{array}\right)-\left(\nu-\rho^{2}\right)\left(\begin{array}{c}
-1 \\
0
\end{array}\right)\right]=\frac{1}{\rho^{2}-\nu-1}\left[\begin{array}{l}
0 \\
y
\end{array}\right]
$$

Thus, the dynamics on the sliding set $\Sigma_{s}$ is governed by the differential equation

$$
\dot{y}=\frac{y}{\rho^{2}-\nu-1},
$$

and as always $\rho^{2}-\nu-1<0$, the pseudo-equilibrium point $(\rho, 0)$ is attractive, behaving as a pseudo-node whenever it exists.

The last step is to analyze the possible boundary equilibrium bifurcation when the parameter $\rho$ is varied. Looking at the admissible equilibria of $\mathbf{f}^{(1)}$, namely $(-\sqrt{\nu}, 0)$ and $(\sqrt{\nu}, 0)$ for $\nu \geq 0$, it is immediate to conclude that we have collisions between these equilibria and the discontinuity boundary for $\rho= \pm \sqrt{\nu}$, or equivalently for the points of the parabola $\nu=\rho^{2}$ in the parameter plane. To apply Theorem 1, we need to check the transversality and the non-degeneracy condition, for which we compute 


$$
\begin{aligned}
& A=D_{\mathbf{x}} \mathbf{f}^{(1)}( \pm \sqrt{\nu}, 0)=\left[\begin{array}{cc}
\mp 2 \sqrt{\nu} & 0 \\
0 & -1
\end{array}\right] \\
& B=\mathbf{f}^{(2)}( \pm \sqrt{\nu}, 0)=\left[\begin{array}{c}
-1 \\
0
\end{array}\right], \quad C^{T}=D_{\mathbf{x}} h( \pm \sqrt{\nu}, 0)=\left[\begin{array}{ll}
1 & 0
\end{array}\right]
\end{aligned}
$$

along with

$$
M=D_{\rho} \mathbf{f}^{(1)}( \pm \sqrt{\nu}, 0)=\left[\begin{array}{l}
0 \\
0
\end{array}\right], \quad \text { and } N=D_{\rho} h( \pm \sqrt{\nu}, 0)=-1 .
$$

We must exclude the case $\nu=0$, the origin of the parameter plane, where $A$ turns out to be singular. For $\nu \neq 0$, we see that $N-C^{T} A^{-1} M=-1$ and the transversality requirement holds. Regarding the non-degeneracy condition, we obtain

$$
C^{T} A^{-1} B=\left[\begin{array}{ll}
1 & 0
\end{array}\right]\left[\begin{array}{cc}
\mp \frac{1}{2 \sqrt{\nu}} & 0 \\
0 & -1
\end{array}\right]\left[\begin{array}{c}
-1 \\
0
\end{array}\right]= \pm \frac{1}{2 \sqrt{\nu}},
$$

so that we will have persistence at the collision for $(\sqrt{\nu}, 0)$ and non-smooth fold at $(-\sqrt{\nu}, 0)$.

The bifurcation set computed above is depicted in Fig. 1 where, apart from the two branches of the parabola corresponding to the two different boundary equilibrium bifurcations, we see the half-straight line $\nu=0$, with $\rho>0$, where a standard saddle-node bifurcation takes place. These lines, organized around the origin which behaves like a codimension two bifurcation point, determine four open regions in the parameter plane. Within each parameter region the phase planes are qualitative similar.

The phase space diagrams corresponding to the various regions labelled in Fig. 1 are shown in Fig. 2. Starting from region 1 in Fig. 2(1), we see that no equilibrium exist. Then, under parameter variation, the bifurcation curve corresponding to a smooth fold bifurcation is crossed as we move into region 2. As shown in Fig. 2(2), this corresponds to the creation of two admissible equilibria. Further parameter variations, cause one of this equilibria to undergo a BEB associated to persistence (as shown analytically above). Hence, in region 3, we see the coexistence of one admissible equilibrium and a pseudo equilibrium (see Fig. 2(3)) which are then annihilated through another BEB along the curve labelled as $E_{A}$ in Fig. 1.

5.2. Case Study 2: Boundary-center bifurcation. As a second example of a possible non-hyperbolic point which collides with the discontinuity surface, let us consider as before that the manifold $\Sigma$ is given by the vertical 
line $x=\rho$, that is $h(x, y)=x-\rho$, so that for $x<\rho$ we have the vector field

$$
\mathbf{f}^{(1)}(x, y)=\left[\begin{array}{c}
\nu x-y \\
x
\end{array}\right] \text {. }
$$

Thus, there is only a possible equilibrium (to be admissible or virtual depending on $\rho)$ at $(\bar{x}, \bar{y})=(0,0)$ that becomes non-hyperbolic for $\nu=0$ and will cross $\Sigma$ for $\rho=0$. Now the non-hyperbolicity comes from the fact that the eigenvalues are on the imaginary axis of the complex plane for critical values of parameters. Thus, the equilibrium is a linear focus as long as $0<|\nu|<2$, being a linear center for $\nu=0$. As the second vector field involved, let us consider again the case of a constant horizontal vector field pushing to the left, say

$$
\mathbf{f}^{(2)}(x, y)=\left[\begin{array}{c}
-1 \\
0
\end{array}\right]
$$

We must anticipate that for $\rho>0$, the passage through the line $\nu=0$ constitutes a bifurcation, since the origin changes it stability becoming a unstable focus for $\nu>0$. When $\nu=0$, we have a linear center configuration bounded by $\Sigma$.

To compute the sliding set $\Sigma_{s}$, we proceed as before by recalling (15), getting the conditions $x=\rho, y-\nu x \leq 0$, and so we arrive at

$$
\Sigma_{s}=\Sigma_{s}(\rho, \nu)=\left\{(x, y) \in \mathbb{R}^{2}: x=\rho, \quad y \leq \nu \rho\right\},
$$

which represents a half part of the straight line $\Sigma$. Note that the boundary of this set is the point $(\rho, \nu \rho)$, where the orbits of $\mathbf{f}^{(1)}$ have a tangency with $\Sigma$, visible for $\rho>0$ and invisible when $\rho<0$. Using GCE condition (16), which reduces here to $x=0$, we detect pseudo-equilibrium points in $\Sigma_{s}$ only if $\rho=0$. Then the system has a continuum of pseudo-equilibria at $(0, y)$ with $y \leq 0$, independently on the value of $\nu$, which leads to a vertical line in the bifurcation set, namely the $\nu$-axis of the parameter plane. Since now

$$
\left\langle\nabla h(\mathbf{x}), \mathbf{f}^{(2)}(\mathbf{x})-\mathbf{f}^{(1)}(\mathbf{x})\right\rangle=[1,0]^{T}\left[\begin{array}{c}
y-\nu x-1 \\
x
\end{array}\right]=y-\nu x-1,
$$

from (6) we get

$$
\begin{aligned}
F_{s}(\mathbf{x}) & =\frac{1}{y-\nu x-1}\left[(-1)\left(\begin{array}{c}
\nu x-y \\
x
\end{array}\right)-(\nu x-y)\left(\begin{array}{c}
-1 \\
0
\end{array}\right)\right]= \\
& =\frac{1}{y-\nu x-1}\left[\begin{array}{c}
0 \\
-x
\end{array}\right] .
\end{aligned}
$$

Thus, the dynamics on the sliding set $\Sigma_{s}$ is governed by the nonlinear differential equation

$$
\dot{y}=\frac{\rho}{1+\nu \rho-y},
$$


so that, as $y \leq \nu \rho$, the sign of $\dot{y}$ coincides with the sign of $\rho$. This dynamics is responsible for creating one stable limit cycle including some upper part of the sliding set, surrounding the unstable focus that exists for $0<\nu,<2$ when $\rho>0$. The bifurcation undergone by the system for $\rho>0$ at $\nu=0$, is reminiscent of the focus-center-limit cycle bifurcation reported in (FreirePonce-Ros 1999), in the sense that the phenomenon is similar, for the limit cycle appears suddenly with significant size, 'coming' from the outermost periodic orbit of the center.

If we want to analyze the boundary equilibrium bifurcation of the origin at $\rho=0$ according to Theorem 1 , we must first to check its hypotheses. Here

$$
A=D_{\mathbf{x}} \mathbf{f}^{(1)}(0,0)=\left[\begin{array}{cc}
\nu & -1 \\
1 & 0
\end{array}\right],
$$

while the other elements are as before, namely

$$
\begin{aligned}
& B=\mathbf{f}^{(2)}(0,0)=\left[\begin{array}{c}
-1 \\
0
\end{array}\right], \quad C^{T}=D_{\mathbf{x}} h(0,0)=\left[\begin{array}{ll}
1 & 0
\end{array}\right], \\
& M=D_{\rho} \mathbf{f}^{(1)}(0,0)=\left[\begin{array}{l}
0 \\
0
\end{array}\right], \quad \text { and } N=D_{\rho} h(0,0)=-1 .
\end{aligned}
$$

We get $N-C^{T} A^{-1} M=-1$, so that the collision is transversal. But, regarding the non-degeneracy condition, we now obtain

$$
C^{T} A^{-1} B=\left[\begin{array}{ll}
1 & 0
\end{array}\right]\left[\begin{array}{cc}
0 & 1 \\
-1 & \nu
\end{array}\right]\left[\begin{array}{c}
-1 \\
0
\end{array}\right]=0,
$$

telling us that the bifurcation is degenerate. In fact, we already know that just in the collision the only equilibrium point that exists for $\rho>0$ exploits in a continuum of pseudo-equilibria, and no equilibria remain for $\rho<0$.

The bifurcation set in $(\rho, \nu)$-plane and the state space diagrams are shown in Fig. 3 and Fig. 4 respectively. In region 1 we have a stable equilibrium of center type which loses its stability in passing to region 2 and gives rise to a stable sliding limit cycle surrounding the unstable equilibrium. This bifurcation is similar the Focus-Centre-Limit Cycle (F-C-LC) bifurcation studied in (Freire, Ponce and Ros, 1999). As mentioned earlier, while a continuum of pseudo-equilibria is detected on the line $\{\rho=0\}$, no equilibria persist for $\rho<0$.

5.3. Case Study 3: Boundary-Hopf Bifurcation. Let us consider a case very similar in principle to the previous one, by substituting only the linear vector field responsible for the non-hyperbolic point and choosing instead the vector field associated to a canonical Hopf singularity, namely

$$
\mathbf{f}^{(1)}(x, y)=\left[\begin{array}{c}
-y+x\left(\nu-x^{2}-y^{2}\right) \\
x+y\left(\nu-x^{2}-y^{2}\right)
\end{array}\right],
$$




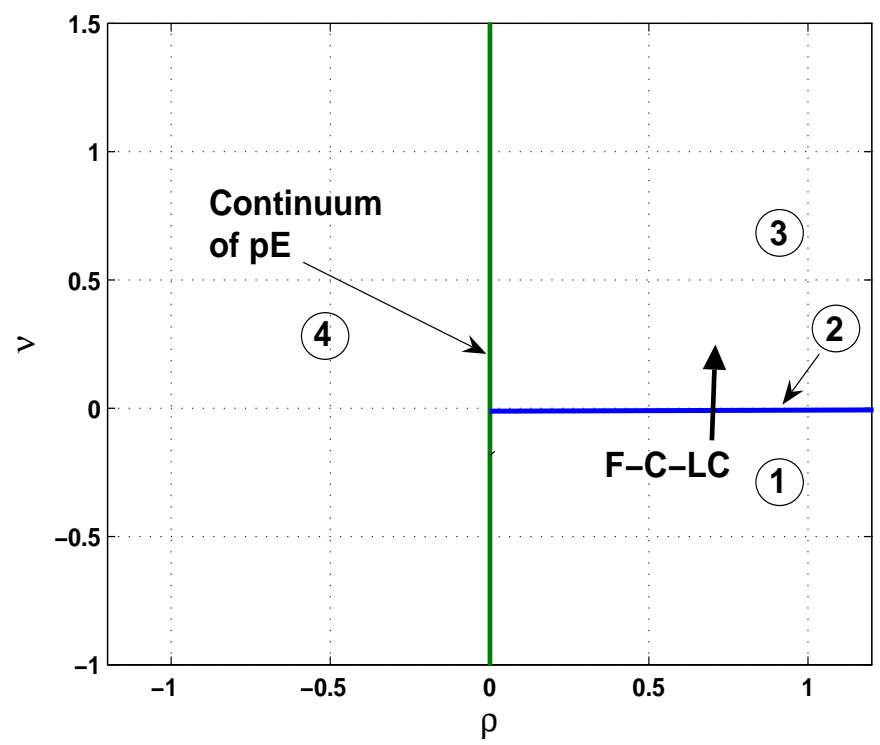

FiguRE 3. Bifurcation set in the plane $(\rho, \nu)$ for the linear center plus a constant vector field case.

which again only have the origin as possible real equilibrium point. We know in advance that for $\rho>0$ and through a Hopf bifurcation at $\nu=0$ the origin passes from being a stable focus to be for $\nu>0$ a unstable focus surrounded by a stable limit cycle, which is a circle of radius $\sqrt{\nu}$.

The sliding set $\Sigma_{s}$ is, by recalling (15), the set of points with $x=\rho, \quad y-$ $x\left(\nu-x^{2}-y^{2}\right) \leq 0$, namely

$$
\Sigma_{s}=\Sigma_{s}(\rho, \nu)=\left\{(x, y) \in \mathbb{R}^{2}: x=\rho, \quad \rho y^{2}+y+\rho\left(\rho^{2}-\nu\right) \leq 0\right\} .
$$

Note that this set is, for $\rho>0$, bounded when non-empty, and unbounded and possibly disconnected for $\rho<0$. If $\rho=0$ the set is the negative $y$-axis. A complete description of the sliding set is given in Tab. 2, where when they are well defined, we introduce the values

$$
y_{l}=-\frac{1}{2 \rho}-\sqrt{\nu-\rho^{2}+\frac{1}{4 \rho^{2}}}, \quad y_{u}=-\frac{1}{2 \rho}+\sqrt{\nu-\rho^{2}+\frac{1}{4 \rho^{2}}} .
$$

Proceeding further, we see that

$$
\left\langle\nabla h(\mathbf{x}), \mathbf{f}^{(2)}(\mathbf{x})-\mathbf{f}^{(1)}(\mathbf{x})\right\rangle=1-y+x\left(\nu-x^{2}-y^{2}\right),
$$


(1)

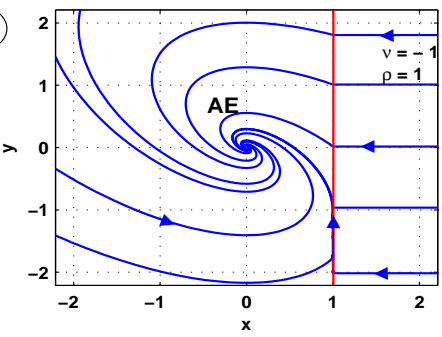

(3)

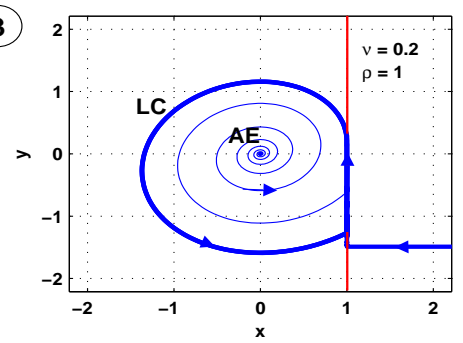

(2)

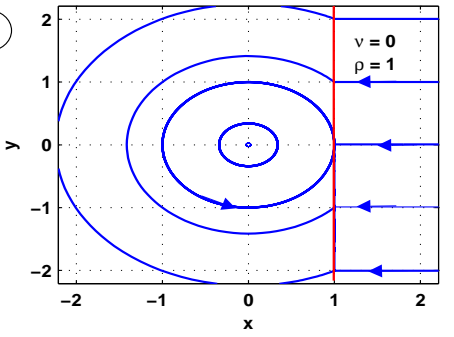

(4)

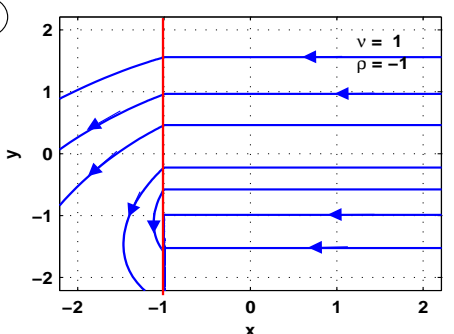

Figure 4. Some state space diagrams in the plane $(x, y)$ for the linear center plus a constant vector field case.

\begin{tabular}{|c|c|}
\hline Parameter region & Sliding part $\Sigma_{s}$ of $\Sigma$ \\
\hline$\rho>0, \quad \nu<\rho^{2}-\frac{1}{4 \rho^{2}}$ & $\emptyset$ \\
\hline$\rho>0, \quad \nu \geq \rho^{2}-\frac{1}{4 \rho^{2}}$ & A bounded segment, with $y_{l} \leq y \leq y_{u}$ \\
\hline$\rho=0$ & The half-axis $y \leq 0$ \\
\hline$\rho<0, \quad \nu>\rho^{2}-\frac{1}{4 \rho^{2}}$ & The two unbounded parts, $y \leq y_{l}$ and $y_{u} \leq y$ \\
\hline$\rho<0, \quad \nu \leq \rho^{2}-\frac{1}{4 \rho^{2}}$ & The complete line $x=\rho$, that is $\Sigma_{s}=\Sigma$ \\
\hline
\end{tabular}

TABLE 2. Definition of the sliding region $\Sigma_{s}$ for various parameter values

and from (6) we get

$$
F_{s}(\mathbf{x})=\frac{1}{y-x\left(\nu-x^{2}-y^{2}\right)-1}\left[\begin{array}{c}
0 \\
x+y\left(\nu-x^{2}-y^{2}\right)
\end{array}\right] .
$$

Thus, the dynamics on the sliding set $\Sigma_{s}$ is governed by the nonlinear differential equation

$$
\dot{y}=\frac{\rho+y\left(\nu-\rho^{2}-y^{2}\right)}{y-\rho\left(\nu-\rho^{2}-y^{2}\right)-1} .
$$


5.3.1. Existence and stability of pseudo-equilibria. Using GCE condition (16), which now is equal to

$$
x+y\left(\nu-x^{2}-y^{2}\right)=0,
$$

we arrive at the condition

$$
q(y):=y^{3}+\left(\rho^{2}-\nu\right) y-\rho=0,
$$

which must be fulfilled by any pseudo-equilibrium candidate, for which we must also check its belonging to $\Sigma_{s}$. Note that, due to the special form of $\mathbf{f}^{(2)}$, this equation corresponds exactly with the horizontal nullcline of $\mathbf{f}^{(1)}$. Equation (20) can have up to three real solutions and the following auxiliary result will be useful.

Lemma 1. For every negative root of (20), the point $(\rho, y)$ belongs to $\Sigma_{s}$, while all positive solutions are such that $(\rho, y)$ is in $\Sigma \backslash \Sigma_{s}$.

Proof. The case $\rho=0$ is direct. Assume $\rho \neq 0$, and let $y$ be a negative root of (20). By multiplying this equation by $\rho$, we can write

$$
0=\rho y^{3}+\rho\left(\rho^{2}-\nu\right) y-\rho^{2},
$$

which is equivalent, after adding $y^{2}$ and arranging terms, to

$$
\rho^{2}+y^{2}=\rho y^{3}+\rho\left(\rho^{2}-\nu\right) y+y^{2}=y\left[\rho y^{2}+y+\rho\left(\rho^{2}-\nu\right)\right] .
$$

Now, since the left hand side is positive and $y<0$ by assumption, we conclude that $\rho y^{2}+y+\rho\left(\rho^{2}-\nu\right)<0$, and so $(\rho, y) \in \Sigma_{s}$. The argument for positive root is completely analogue and the lemma follows.

The number of pseudo-equilibria could change by moving parameters. In particular, from the above lemma, we should study the possible change in the number of negative solutions of (20). By computing its derivative, we obtain

$$
q^{\prime}(y)=3 y^{2}+\rho^{2}-\nu
$$

If for instance $\rho^{2}>\nu$, then the function $q(y)$, is increasing, having only one real root, since its derivative is always positive. If furthermore $\rho>0$, such root is positive as $q(0)=-\rho<0$, and then there are no pseudo-equilibria, according to the lemma.

Eq. (20) will start to have negative roots if we choose the parameters to get a maximum in a point $y^{*}<0$ where both $q\left(y^{*}\right)=0$ and $q^{\prime}\left(y^{*}\right)=0$, for then the graph of $q$ in the plane $(y, q)$ has a tangency with the negative horizontal axis. The existence of such a point will imply a saddle-node bifurcation of pseudo-equilibria $(\mathrm{SNpE})$, for then a change of parameters should lead to the appearance of two negative roots for (20).

Thus, we will eliminate $y$ in the system formed by the two equations $q(y)=0, q^{\prime}(y)=0$, trying to obtain a parameter relation that corresponds 
with the SNpE bifurcation. From $q^{\prime}(y)=0$, we obtain the relation $\nu=$ $3 y^{2}+\rho^{2}$, and substituting in (20), we get

$$
-2 y^{3}-\rho=0 .
$$

By assuming $\rho>0$, we have

$$
y^{*}=\left(-\frac{\rho}{2}\right)^{\frac{1}{3}}
$$

and replacing this value in $\nu=3 y^{2}+\rho^{2}$, we finally arrive at the condition

$$
\nu=\rho^{2}+3\left(\frac{\rho}{2}\right)^{2 / 3} \text {. }
$$

This curve in the parameter plane $(\rho, \nu)$ determines where the $\mathrm{SNpE}$ takes place.

The phenomenon is associated to the fact that the boundary $\Sigma$ intersects the horizontal nullcline in two points, after becoming tangent to it at the point $(x, y)=\left(\rho, y^{*}\right)$. If we check the sign of $\dot{y}$ on $\Sigma_{s}$, we observe that it coincides with the sign of $q(y)$. Therefore, it is immediate to conclude that after the $\mathrm{SNpE}$ bifurcation the upper pseudo-equilibrium behaves as a saddle while the lower one as a stable node.

Note that, when this tangency between the nullcline and the boundary is detected, the limit cycle, surrounding the unstable equilibrium, cannot continue to exist. In particular, for the cycle to exist past this tangency, it should develop a sliding segment lying on the vertical line $x=\rho$. Such a sliding segment should be confined to the region given by

$$
-3\left(\frac{\rho}{2}\right)^{2 / 3} \leq y \leq 3\left(\frac{\rho}{2}\right)^{2 / 3} .
$$

Such region, though, certainly contains the point $y^{*}$, where two pseudoequilibria are created at the tangency. So, clearly the cycle cannot coexist with such new invariant sets and therefore ceases to exist.

The two pseudo-equilibria will exist for

$$
\nu>\rho^{2}+3\left(\frac{\rho}{2}\right)^{2 / 3}
$$

provided that $\rho>0$; if we let $\rho$ tend to zero then from (20) we deduce that the stable pseudo-node tends to $(0, \sqrt{\nu})$ while the pseudo-saddle tends to the origin where we will have a boundary equilibrium collision.

5.3.2. $B E B$ of the origin. Let us finally analyze the boundary equilibrium bifurcation of the origin at $\rho=0$. As before, $M$ vanishes and $N=D_{\rho} h(0,0)=$ 
-1 , so that the transversality condition is satisfied. To check the nondegeneracy condition of Theorem 1 , we see now

$$
\begin{aligned}
C^{T} A^{-1} B & =\left[\begin{array}{ll}
1 & 0
\end{array}\right]\left[\begin{array}{cc}
\nu & -1 \\
1 & \nu
\end{array}\right]^{-1}\left[\begin{array}{c}
-1 \\
0
\end{array}\right]= \\
& =\frac{1}{1+\nu^{2}}\left[\begin{array}{ll}
1 & 0
\end{array}\right]\left[\begin{array}{cc}
\nu & 1 \\
-1 & \nu
\end{array}\right]\left[\begin{array}{c}
-1 \\
0
\end{array}\right]=\frac{-\nu}{1+\nu^{2}}
\end{aligned}
$$

so that depending on the sign of $\nu$ we have a different kind of boundary bifurcation. Thus, for $\nu<0$ we have persistence or transition between one real equilibrium and one pseudo-equilibrium, whilst for $\nu>0$ there is a non-smooth fold between them.

5.3.3. Grazing bifurcation. We also must take into account that through a Hopf bifurcation at $\nu=0$ for $\rho>0$, the origin becomes surrounded for $\nu>0$ by a stable limit cycle, namely a circle of radius $\sqrt{\nu}$. We could speak so of a grazing bifurcation when the discontinuity manifold $\Sigma$ touches the limit cycle. Clearly, it happens for $\rho>0$ at the curve $\nu=\rho^{2}$ of the plane $(\rho, \nu)$. Apart from the appearance of a sliding segment in the cycle, there are no other qualitative changes. Much more dramatic is the change that occurs by crossing the curve associated to the $\mathrm{SNpE}$ bifurcation; the stable limit cycle is firstly transformed in a loop containing the saddle-node point, and after crossing the SNpE curve is to be broken in different orbits, so that from all initial conditions, excepting the origin and the pseudo-saddle, the orbits tend to approach (in finite time) the stable pseudo-node. A new almost global attractor is then created.

Putting all this information together, we show in Fig. 5 the bifurcation set in the plane $(\rho, \nu)$. The number and types of invariant sets existing in each of the regions in the two-parameter space is given in Tab. 3. Starting from the bottom right-hand region of the diagram in Fig. 5, we notice the occurrence of a Hopf bifurcation as we cross into region 1. Here an unstable admissible equilibrium coexist with a stable limit cycle. Under parameter variations, as we enter region 2 , the cycle undergoes a grazing bifurcation which gives rise a stable sliding limit cycle. Then, because of a saddle-node of pseudo-equilibria, in region 3 we observe the coexistence of one unstable admissible equilibria and a pair of pseudo-equilibria (a pseudo-saddle and a pseudo-node). The stable pseudo-saddle then collides on the boundary with the unstable admissible equilibria so that through a nonsmooth-fold associated to the BEB they both disappear. Hence in region 4, we have only one stable pseudo-node. Such a node undergoes another BEB along the curve labelled as $E_{T}$ in Fig. 5 which give rise to a stable admissible equilibrium in region 0 . 


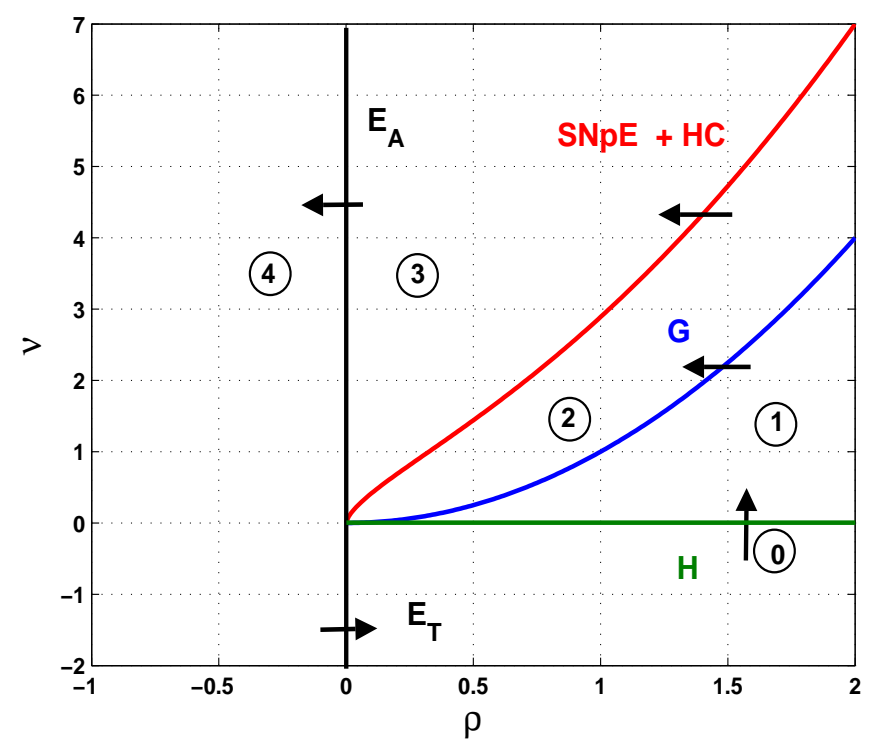

Figure 5. Bifurcation set in the plane $(\rho, \nu)$ for the Hopf + constant vector field case.

\begin{tabular}{|c|c|}
\hline Region & Invariant sets \\
\hline 0 & 1 stable AE \\
\hline 1 & 1 unstable AE +1 sLC \\
\hline 2 & 1 unstable AE +1 sLC with a sliding segment \\
\hline 3 & 1 unstable AE +1 pseudo-saddle +1 stable pseudo-node \\
\hline 4 & 1 stable pseudo-node \\
\hline
\end{tabular}

TABLE 3. Invariant sets in each of the regions depicted in

Fig. 5

5.4. Discussion. The three case studies investigated above show some general features that we conjecture can be observed also in higher-dimensional Filippov systems, undergoing similar types of boundary-equilibrium bifurcations.

In particular, in all cases the unfolding of the two-parameter BEB point shows nearby bifurcation curves of two types: (i) curves associated to the specific type of smooth bifurcation exhibited by the system away from the 

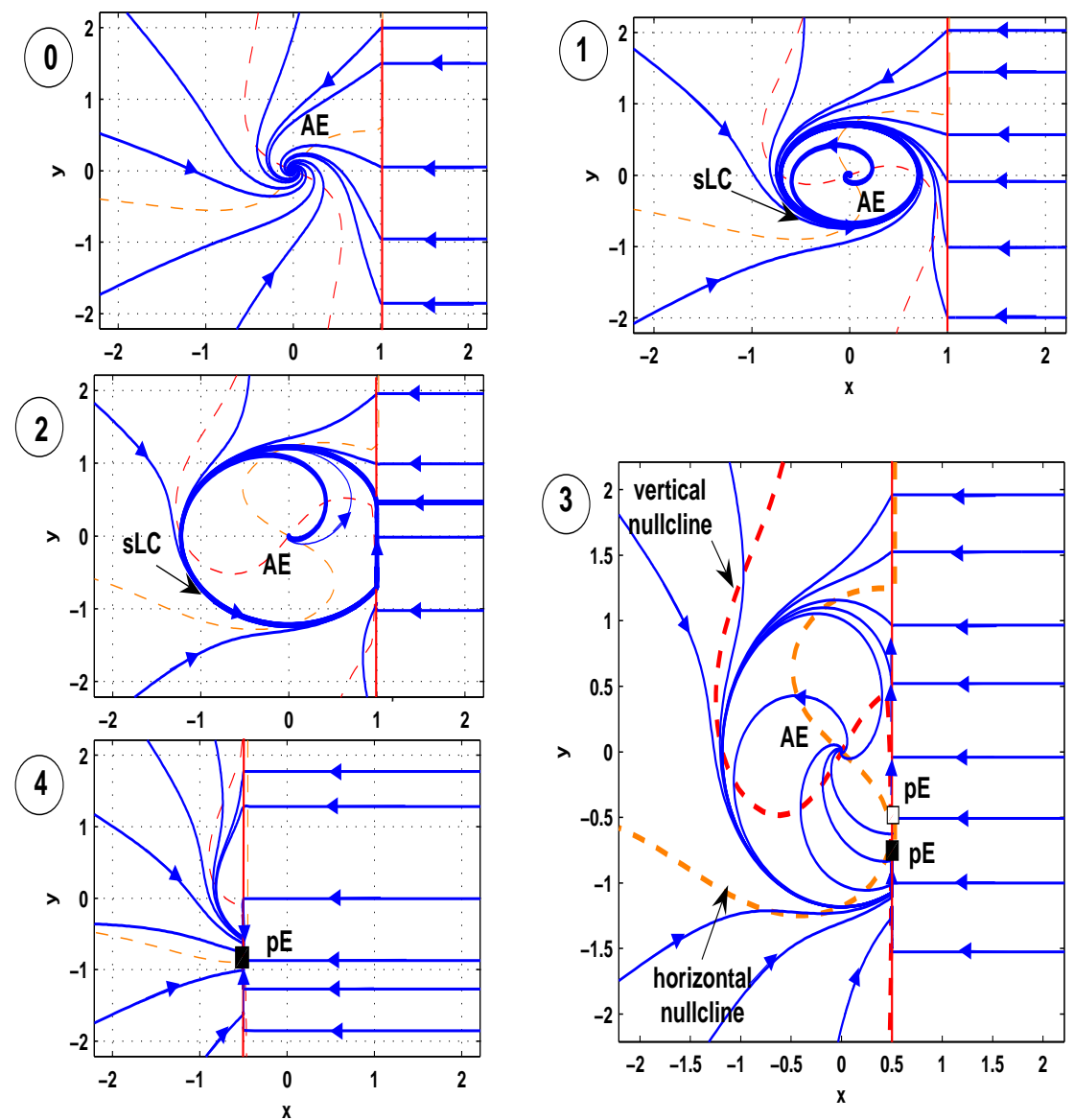

Figure 6. State space diagrams in the plane $(x, y)$ for Hopf + constant vector field case.

boundary; and (ii) curves corresponding to discontinuity-induced bifurcations of the invariant sets branching off from such smooth bifurcation points. For example, in the boundary-fold case, we detect the saddle-node bifurcation of the smooth part of the vector field giving rise to a stable and an unstable equilibrium. We can then expect that, even in higher-dimensional systems, the two equilibria branching off the smooth saddle-node can then undergo BEB nearby as another parameter of the system is varied. Thus, we conjecture that a local picture similar to the one shown in Fig. 1. will also be found in higher-dimensional examples. 
A similar argument will hold for the other two cases. In the boundaryHopf, for instance, we conjecture that the limit cycle branching off the smooth Hopf bifurcation point will generically undergo a grazing bifurcation nearby. Therefore, we can expect the local unfolding of the two-parameter boundary-Hopf bifurcation to involve at least two bifurcation curve, in the simplest case: the curve of smooth Hopf bifurcations and the curve of the grazing of the ensuing limit cycle. This will give rise to a local bifurcation diagram similar to the one depicted in Fig. 5.

Another general feature that we observed in the unfoldings presented in this paper is that, except for the degenerate case of boundary center, the BEB curves near the two-parameter bifurcation point are associated to both persistence and non-smooth fold scenarios.

To summarize, we believe that the results presented in this paper and the case studies we analyze are representative of phenomena that will be also observed in higher-dimensions. The investigation of such phenomena is the subject of ongoing research.

\section{Conclusions}

A two-parameter methodology has been developed to analyze non-hyperbolic boundary equilibrium bifurcations starting from a thoroughly review of the one-parameter framework. The main non-hyperbolic planar cases, namely the one-zero eigenvalue, the linear and nonlinear imaginary eigenvalue pair situations have been considered when on the other side of the discontinuity manifold a constant vector field is applied.

It is worth mentioning here that the representative cases which were studied in the paper can be particularly relevant to study the dynamics of switched control systems close to some smooth bifurcation of interest. For example, if a sliding control strategy is used to stabilize the equilibrium point of some system of interest, then the closed loop system typically has a boundary equilibrium point. Our strategy explains the simplest scenarios that can occur when such a point becomes non-hyperbolic under parameter variations.

Future work will address the generalization of the case studies presented above to higher-dimensional Filippov systems with more general vector fields. In particular, it seems interesting to assume a re-injecting nonconstant vector field for the three cases analyzed in the paper. Preliminary work on this subject suggests that the bifurcation sets can change dramatically, even when the 'right' vector field does not introduce new admissible equilibria. 


\section{ACKNOWLEDGEMENTS}

The authors wish to thank the Centre de Recerca Matematica (CRM) and the Government of Catalunya for their support. The paper was completed during a research visit of the authors at the CRM in Barcelona in March 2007. D.J. Pagano acknowledges CNPq/Brazil for funding its work under grant PDE-202218/2006-6. E. Ponce is partially supported by grant MTM2006-00847 from Spanish Ministery of Educación y Ciencia.

\section{REFERENCES}

Banerjee, S. and Verghese, G. C. (2001). Nonlinear phenomena in power electronics, IEEE Press.

Brogliato, B. (1999). Nonsmooth Mechanics - Models, Dynamics and Control, SpringerVerlag, New York.

Brogliato, B. (2000). Impacts in Mechanical Systems - Analysis and Modelling, SpringerVerlag, New York. Lecture Notes in Physics, Volume 551.

di Bernardo, M., Budd, C., Champneys, A., Kowalczyk, P., Nordmark, A., Olivar, G. and Piiroinen, P. (2006). Bifurcations in nonsmooth dynamical systems, SIAM Review, to appear.

di Bernardo, M., Johansson, K. H. and Vasca, F. (2001). Self-Oscillations and Sliding in Relay Feedback Systems: Symmetry and Bifurcations, International Journal of Bifurcations and Chaos 11(4): 1121-1140.

di Bernardo, M., Kowalczyk, P. and Nordmark, A. (2002). Bifurcations of dynamical systems with sliding: derivation of normal-form mappings, Physica D 170: 175-205.

di Bernardo, M., Nordmark, A. and Olivar, G. (2007). Discontinuity-induced bifurcations of equilibria in piecewise-smooth dynamical systems, submitted to Physica D.

Cunha, F.B., Pagano, D.J. and Moreno, U.F. (2003). Sliding bifurcations of equilibria in planar variable structure systems, IEEE Trans. Circuits and Systems I 50(8): 1129$\underline{1134}$.

Filippov, A. F. (1988). Differential Equations with Discontinuous Righthand Sides, Kluwer Academic Publishers, Dortrecht.

Freire, E., Ponce, E. and Ros, J. (1999). Limit cycle bifurcation from center in symmetric piecewise-linear systems, International Journal of Bifurcation and Chaos 9(5): 895907.

Garcia, M., Chatterjee, A., Ruina, A. and Coleman, M. (1998). The Simplest Walking Model: stability, complexity and scaling, ASME Journal of Biomechanical Engineering 120: 281-288.

Kowalczyk, P., di Bernardo, M., Champneys, A., Hogan, S., Homer, M., Kuznetsov, Y., Nordmark, A. and Piiroinen, P. (2006). Two-parameter nonsmooth bifurcations of limit cycles: classification and open problems, International Journal of Bifurcations and Chaos 6: 601-629.

Kuznetsov, Y. A., Rinaldi, S. and Gragnani, A. (2003). One-parameter bifurcations in planar Filippov systems, International Journal of Bifurcations and Chaos 13(8): 2157$\underline{2188 .}$.

MARIO Di BERnARdo,

Dept. of Systems and Computer Science, University of Naples Federico II,

Naples, Italy and Dept. of Engineering Maths, University of Bristol, U.K. 
DANiel J. Pagano,

Dept. of Engineering Maths, University of Bristol, UK; on leave from Dept. of Automation and Systems, UFSC, Brazil

ENRIQUe Ponce,

Dept. Applied Maths, ets Ingenieros, Universidad de Sevilla, Camino de los Descubrimientos Sn, 41092-Sevilla Spain, eponcem@us.es 